\title{
On the Stability of the Ritz-Galerkin Method for Hammerstein Equations
}

\author{
by Jörg Hertling and Alexandru I. Şchiop
}

\begin{abstract}
For the numerical treatment of Hammerstein equations by variational methods which has been considered by Hertling, we establish the stability in the sense of Mikhlin, Stetter and Tucker.
\end{abstract}

Introduction. If one uses a variational method for the numerical treatment of Hammerstein equations, one obtains a nonlinear algebraic system of equations. In order to investigate the stability of the computing scheme, we will show that one can apply a theorem by Tucker [7]. Tucker's work is based on a paper by Mikhlin [3]. We would also like to refer to a paper by Kasriel and Nashed [2] where the problem of stability has been considered in a very similar way for some classes of nonlinear operator equations.

An equivalent general concept of stability and its application to initial-value problems has been given by Stetter [6].

Let $B$ be a bounded measurable set in a finite-dimensional Euclidean space and let the symmetric kernel $K(x, y)$ define an operator $\mathbf{A}$ which is selfadjoint in $\mathbf{L}^{2}$ and completely continuous from $\mathbf{L}^{q}$ into $\mathbf{L}^{p}\left(p \geqslant 2, p^{-1}+q^{-1}=1\right)$ :

$$
\mathbf{A} u \equiv \int_{B} K(x, y) u(y) d y .
$$

Furthermore, we introduce the Nemytsky operator

$$
\mathbf{h} \equiv g(u(y), y)
$$

as a continuous operator from $\mathbf{L}^{p}$ into $\mathbf{L}^{q}$; we assume that $g(u, y)$ is an $N$-function and that $\mathbf{h}$ is potential. A function $g(u, y)$ is an $N$-function if it is continuous with respect to $u$ for almost every $y \in B$ and measurable in $B$ with respect to $y$ for every fixed $u \in$ $(-\infty,+\infty)$. An operator $h$ from a Banach space $\mathbf{E}$ into the conjugate space $\mathbf{E}^{*}$ is called potential on some set $H \subset \mathbf{E}$, if there exists a functional $f$ such that $\operatorname{grad} f(x)=\mathbf{h}(x)$ for every $x \in H$. Let $G(u, y)$ be defined by

$$
\partial G(u, y) / \partial u \equiv g(u, y)
$$

and assume

Received August 27, 1973, revised November 29, 1973 and June 4, 1974.

AMS (MOS) subject classifications (1970). Primary 45 L10, 65R05; Secondary 65N30.

Key words and phrases. Hammerstein equation, Ritz-Galerkin method, stability of the numerical computation, finite element method. 


$$
G(0, y) \equiv 0
$$

For the Hammerstein equation

$$
u=\mathbf{A h} u,
$$

one of the authors [1] has considered the numerical solution by means of a Ritz-Galerkin scheme and by using subspaces of spline functions and finite elements. We shall establish here the stability of this approximating scheme.

The Computing Scheme and its Stability. According to Vainberg [8], there holds the following

TheORem 1. Let $\mathbf{A}$ be positive and

$$
2 G(u, y) \leqslant a u^{2}+b(y)|u|^{\alpha}+c(y),
$$

where $0 \leqslant a \leqslant \lambda_{1}\left(\lambda_{1}\right.$ is the smallest characteristic number of $\left.\mathbf{A}\right), 0<\alpha<2,0 \leqslant$ $b(y) \in \mathbf{L}^{\gamma}, \gamma=2 /(2-\alpha), 0 \leqslant c(y) \in \mathbf{L}$. Then, Eq. (1.5) has at least one solution in $\mathbf{L}^{p}$. If, in addition, $\mathbf{h}$ satisfies a Lipschitz condition

$$
\left\|\mathrm{h} u_{2}-\mathrm{h} u_{1}\right\|_{\mathrm{L} q} \leqslant C\left\|u_{2}-u_{1}\right\|_{\mathrm{L}},
$$

then the solution is unique.

Henceforth, we shall assume (2.2). The proof of this theorem consists in minimizing the functional

$$
\varphi(u)=(u, u)-2 f\left(\mathbf{A}^{1 / 2} u\right)
$$

in $L^{2}$, where $A^{1 / 2}$ is completely continuous from $L^{2}$ into $L^{p}$ and

$$
f(u)=\int_{B} G(u(y), y) d y .
$$

Since $\operatorname{grad} f(u)=\mathrm{h} u$, the minimization of (2.3) yields a solution $u_{0} \in \mathrm{L}^{2}$ of

$$
u=\mathbf{A}^{1 / 2} \mathbf{h} \mathbf{A}^{1 / 2} u ;
$$

setting $z_{0}=\mathrm{A}^{1 / 2} u_{0}$, we have a solution of (1.5). The Lipschitz condition implies that $u_{0}$ strictly minimizes the functional $(2.3)$ in $\mathbf{L}^{2}$;

For some $u_{1}, u_{2} \in \mathbf{L}^{2}$, it follows from (2.2):

$$
\begin{aligned}
\| \operatorname{grad} & \varphi\left(u_{2}\right)-\operatorname{grad} \varphi\left(u_{1}\right) \| \\
& \geqslant 2\left\|u_{2}-u_{1}\right\|-2\left\|\mathbf{A}^{1 / 2} \mathbf{h} \mathbf{A}^{1 / 2} u_{2}-\mathbf{A}^{1 / 2} \mathbf{h} \mathbf{A}^{1 / 2} u_{1}\right\| \\
& \geqslant 2\left\|u_{2}-u_{1}\right\|-2 \lambda_{1}^{-1 / 2}\left\|\mathbf{h} \mathbf{A}^{1 / 2} u_{2}-\mathbf{h A}^{1 / 2} u_{1}\right\|_{\mathbf{L}} q \\
& \geqslant 2\left(1-C / \lambda_{1}\right)\left\|u_{2}-u_{1}\right\| .
\end{aligned}
$$

For the numerical approximation, we consider the minimization of $\varphi(w)$ on a finitedimensional subspace $\mathbf{L}_{m}^{2}$ of $\mathbf{L}^{2}$ with $\operatorname{dim}\left(\mathbf{L}_{m}^{2}\right)=m$. Let $\mathbf{L}_{m}^{2}$ be spanned by the functions $\left\{w_{i}(y)\right\}_{i=1}^{m}$. If we represent a function in $\mathrm{L}_{m}^{2}$ by $\sum_{i=1}^{m} u_{i} w_{i}(y)$ and if we define $\varphi\left(\Sigma_{i=1}^{m} u_{i} w_{i}(y)\right) \equiv G\left(u_{1}, \cdots, u_{m}\right) \equiv G(\mathbf{u})$, then it has been shown in [1] 
that there exists a positive constant $C_{1}$ such that

$$
G(\mathbf{u}) \geqslant \varphi\left(u_{0}\right)+\frac{\lambda_{1}-C}{\lambda_{1} C_{1}^{2}} \sum_{i=1}^{m}\left|u_{i}\right|^{2}
$$

which entails

$$
\lim _{\|\mathbf{u}\| \rightarrow \infty} G(\mathbf{u})=+\infty .
$$

Since $G(\mathbf{u})$ is continuous on $\mathbf{R}^{m}$, bounded below by $\varphi\left(u_{0}\right)$ and satisfies (2.7), it follows that there exists at least one vector $\hat{\mathbf{u}} \in \mathbf{R}^{m}$ such that $G(\mathbf{u}) \geqslant G(\hat{\mathbf{u}})$ for all $\mathbf{u} \in \mathbf{R}^{m}$.

In order to show that $\hat{\mathbf{u}}$ is unique, one considers

$$
\operatorname{grad} G(\mathbf{u})=2\left(\sum_{j=1}^{m} u_{j} w_{j}\right)-2 \mathbf{A}^{1 / 2} \mathbf{h}\left(\sum_{j=1}^{m} u_{j} \mathbf{A}^{1 / 2} w_{j}\right)=0 .
$$

Applying $\mathbf{A}^{1 / 2}$ to this equation and denoting $\bar{w}_{j}=\mathbf{A}^{1 / 2} w_{j}$ yields

$$
\sum_{j=1}^{m} u_{j} \bar{w}_{j}=\mathrm{Ah}\left(\sum_{j=1}^{m} u_{j} \bar{w}_{j}\right)
$$

From (2.2), we obtain that $\mathbf{A h}$ is a contracting mapping with a unique fixed point $\hat{\mathbf{u}}$. This means that there exists a unique function $\hat{w}_{m}$ in the subspace $\mathbf{L}_{m}^{2}$ which minimizes the functional (2.3) over $\mathbf{L}_{m}^{2}$.

By applying $A$ to (2.8), we obtain the system

$$
\sum_{j=1}^{m} u_{j}\left(\bar{w}_{j}, \bar{w}_{i}\right)=\left(\mathbf{A h}\left(\sum_{j=1}^{m} u_{j} \bar{w}_{j}\right), \bar{w}_{i}\right), \quad i=1,2, \cdots, m,
$$

which might be solved by some iterative method. The approximate solution of the integral equation is given by

$$
\hat{w}_{m}=\sum_{j=1}^{m} \hat{u}_{j} \bar{w}_{j}
$$

We will denote the system (2.9) by

$$
T_{m} u_{m}=0 \text {. }
$$

Definition 1 [7]. An operator $A_{m}$ is said to lie in an $\Omega_{m}=\left(u_{m}, r_{m}, b_{m}\right)$ neighborhood of an operator $T_{m}$ if $A_{m}=T_{m}+b_{m} U_{m}$, where $U_{m}$ are nonexpansive mappings $\left(\left\|U_{m}(x)-U_{m}(y)\right\|_{m} \leqslant\|x-y\|_{m}\right.$ for all $x, y \in \mathbf{R}^{m},\|\cdot\|_{m}$ denotes the Euclidean norm) in $K_{m}\left(u_{m}, r_{m}\right)=\left\{u \mid\left\|u-u_{m}\right\|_{m} \leqslant r_{m}\right\}$ and $\left\|U_{m} u_{m}\right\|_{m} \leqslant\left\|u_{m}\right\|_{m}$ independently of $m$.

Let the corresponding perturbated Ritz-Galerkin system be

$$
A_{m} v_{m}=\delta_{m}
$$

Definition 2 [7]. The computing scheme (2.11) is stable at $\left\{u_{m}\right\}$ if for each $r_{m}$ there exist neighborhoods $V_{m}\left(0, \eta_{m}\right)$, numbers $p_{m}$ and constants $s$ and $t$ such that, if $A_{m}$ is in an $\Omega_{m} \equiv\left(u_{m}, r_{m}, b_{m}\right)$ neighborhood of $T_{m}$ with $b_{m} \leqslant p_{m}$ and $\delta_{m} \in V_{m}$, 
then Eqs. (2.12) are solvable and

$$
\left\|v_{m}-u_{m}\right\|_{m} \leqslant s b_{m}+t\left\|_{m}\right\|_{m},
$$

where $s$ and $t$ are independent of $n$ (but may depend on the sequence $\left\{u_{m}\right\}$ ).

Now we have the following result:

THEOREM 2. For the construction of the solution, use a subspace $\mathbf{L}_{m}^{2}$ which has the properties

$$
\lim _{m \rightarrow \infty} \inf _{\widetilde{w} \in \mathrm{L}_{m}^{2}}\left\|\widetilde{w}-u_{0}\right\|_{\mathrm{L}^{2}}=0
$$

and strong minimality in the sense of [4]. Then, the computing scheme (2.11) is stable at $\left\{u_{m}\right\}$.

Proof. Using relation (2.6) and strong minimality, it follows that there exists a constant $C_{2}>0$ which is independent of $m$, such that we have for $u, v \in \mathbf{R}^{m}$

$$
\left\|T_{m} u-T_{m} v\right\|_{m} \geqslant C_{2}\|u-v\|_{m} .
$$

On the other hand, the $\left\|u_{m}\right\|_{m}$ are bounded above, independently of $m$ (uniformly bounded above).

Indeed, with our assumptions, we have the following chain of inequalities (see [1]):

$$
\begin{aligned}
& \left(\lambda_{1}-C\right)\left\|\mathbf{A}^{1 / 2}\left(\hat{w}_{m}-u_{0}\right)\right\|_{\mathrm{L}^{2}}^{2} \leqslant\left(1-C / \lambda_{1}\right)\left\|\hat{w}_{m}-u_{0}\right\|_{\mathrm{L}^{2}}^{2} \\
& \leqslant \varphi\left(\hat{w}_{m}\right)-\varphi\left(u_{0}\right)=\inf _{w \in \mathrm{L}_{m}^{2}} \varphi(w)-\varphi\left(u_{0}\right) \\
& \quad \leqslant \inf _{w \in \mathrm{L}_{m}^{2}}\left(\left\|w-u_{0}\right\|_{\mathrm{L}^{2}}^{2}+C \|_{\left.\mathbf{A}^{1 / 2}\left(w-u_{0}\right) \|_{\mathrm{L}^{2}}^{2}\right)}\right. \\
& \quad \leqslant\left(1+\frac{C}{\lambda_{1}}\right) \inf _{w \in \mathrm{L}_{m}^{2}}\left\|w-u_{0}\right\|_{\mathrm{L}^{2}}^{2} \leqslant\left(1+\frac{C}{\lambda_{1}}\right)\left\|_{\widetilde{w}_{m}}-u_{0}\right\|_{\mathrm{L}^{2}}^{2} \\
& \leqslant \frac{\lambda_{1}+C}{\lambda_{1}^{2}}\left\|\mathbf{A}^{1 / 2}\left(\widetilde{w}_{m}-u_{0}\right)\right\|_{\mathrm{L}^{2}}^{2},
\end{aligned}
$$

where $u_{0}$ is the solution of $(2.5), \hat{w}_{m}$ the unique function which minimizes the functional (2.3) over $\mathbf{L}_{m}^{2}$ and $\widetilde{w}_{m}$ the interpolation of $u_{0}$ in $\mathbf{L}_{m}^{2}$. If $\left\|u_{m}\right\|_{m}$ are not uniformly bounded, then we have from (2.7), $\lim _{m \rightarrow \infty} \varphi(\hat{w})=+\infty$, which contradicts the combination of (2.16) and (2.14). Tucker has proved [7] that the uniform boundedness of $\left\{\left\|u_{m}\right\|_{m}\right\}$, together with (2.15), ensures that the computing scheme (2.11) is stable. Q.E.D.

Let us remark that we did not use the existence of the second derivative of the functional (2.3) as has been done by Mikhlin [3] and Schiop [5]. On the other hand, we have to assume (2.14).

Several classes of interpolating functions do, in fact, satisfy this property. In the one-dimensional case, we refer in particular to $L$-splines and their generalizations, in 
the multidimensional case, we refer to finite elements. Most of these constructions have been considered by Varga [9].

Let us finally say that, with the machinery of [1], an analogous proof for the stability of the computing scheme for Hammerstein equations with quasi-definite kernels can be given.

Institut für Numerische Mathematik

Technische Hochschule Wien

Gusshausstr. 27-29

A-1040 Wien, Austria

Institute of Mathematics

Romanian Academy of Sciences

Calea Grivitei

Bucarest 12, Romiania

1. J. HERTLING, Numerical Treatment of Hammerstein-Equations by Variational Methods, R. Ansorge \& W. Törnig (Eds.), Numerische Lösung nichtlinearer partieller Differential- und Integrodifferentialgleichungen, Lecture Notes in Math., no. 267, Springer-Verlag, Berlin, 1972, pp. 267-288.

2. R. H. KASRIEL \& M. Z. NASHED, "Stability of solutions of some classes of nonlinear operator equations," Proc. Amer. Math. Soc., v. 17, 1966, pp. 1036-1042. MR 33 \#7896.

3. S. G. MIKHLIN, “On the stability of certain computational processes," Dokl. Akad. Nauk SSSR, v. 157, 1964, pp. 271-273 = Soviet Math. Dokl., v. 5, 1964, pp. 931-933. MR 31 \#6361.

4. S. G. MIKHLIN, The Numerical Performance of Variational Methods, Wolters-Noordhoff, Groningen, 1971. MR 43 \#4236.

5. A. I. SCHIOP, "Stability of Ritz procedure for nonlinear two-point boundary value problem," Numer. Math., v. 20, 1973, pp. 208-212.

6. H. J. STETTER, Analysis of Discretization Methods for Ordinary Differential Equations, Springer-Verlag, Berlin, 1973.

7. T. S. TUCKER, "Stability of nonlinear computing schemes," SIAM J. Numer. Anal., v. 6, 1969, pp. 72-81. MR 40 \#3743.

8. M. M. VAINBERG, Variational Methods for the Study of Nonlinear Operators, HoldenDay, San Francisco, Calif., 1964. MR 31 \#638.

9. R. S. VARGA, Functional Analysis and Approximation Theory in Numerical Analysis, Regional Conference Series in Applied Mathematics, vol. 3, Soc. Indust. Appl. Math., Philadelphia, Pa., 1971. 